\title{
NORMAL CURVATURE OF SURFACES IN SPACE FORMS
}

\section{IRWen VAlle GUAdalupe AND Lucio RodRiguez}

\begin{abstract}
Using the notion of the ellipse of curvature we study compact surfaces in high dimensional space forms. We obtain some inequalities relating the area of the surface and the integral of the square of the norm of the mean curvature vector with topological invariants. In certain cases, the ellipse is a circle; when this happens, restrictions on the Gaussian and normal curvatures give us some rigidity results.
\end{abstract}

1. Introduction. We consider immersions $f: M \rightarrow Q_{c}^{n}$ of surfaces into spaces of constant curvature $c$. We are going to relate properties of the mean curvature vector $H$ and of the normal curvature $K_{N}$ to geometric properties, such as area and rigidity of the immersion. We use the notion of the ellipse of curvature studied by Little [10], Moore and Wilson [11] and Wong [12]. This is the subset of the normal space defined as $\left\{\alpha(X, X): X \in T_{p} M,\|X\|=1\right\}$, where $\alpha$ is the second fundamental form of the immersion and \|\| is the norm of the vectors. Let $\chi(M)$ denote the Euler characteristic of the tangent bundle and $\chi(N)$ denote the Euler characteristic of the plane bundle when the codimension is 2 . We prove the following generalization of a theorem of Wintgen [13].

THEOREM 1. Let $f: M \rightarrow Q_{c}^{n}$ be an isometric immersion of a compact oriented surface into an orientable n-dimensional manifold of constant curvature $c$. We have the following

$$
\int_{M}\|H\|^{2} d M+c \operatorname{Area}(M) \geq 2 \pi \chi(M)+\left|\int_{M} K_{N} d M\right|
$$

with equality if and only if $K_{N}$ does not change sign and the ellipse of curvature is a circle at every point. If in addition $M$ is homeomorphic to the 2-sphere $S^{2}, n=4$, and $H$ is parallel, then

$$
\left(\|H\|^{2}+c\right) \operatorname{Area}(M)=2 \pi(\chi(M)+|\chi(N)|) .
$$

COROLlary 1. Let $M$ be homeomorphic to the 2-sphere $S^{2}$ and minimal into the 4-sphere $S^{4}(1)$. Then

$$
\operatorname{Area}(M)=2 \pi(\chi(M)+|\chi(N)|) .
$$

Consequently $\operatorname{Area}(M)$ is a multiple of $4 \pi$. Also, two minimal immersions

The first author's work was done under partial support by FAPESP, Proc. No. 80/0251-8, Brasil, and the second author's work was done under partial support by CNPq, Brasil. 
have the same area if and only if they are regularly homotopic (see Barbosa [3]).

COROLlaRY $2^{\dagger}$. Let $M$ be a compact oriented surface immersed into $R^{4}$. Then, if $K_{N}>0$ at every point, we have

$$
\int_{M}\|H\|^{2} d M \geq 12 \pi
$$

The equality holds if and only if the ellipse of curvature is a circle.

Remarks. 1. One way to obtain examples of immersed surfaces that satisfy (1.3) is to take a minimal immersion of the sphere $S^{2}$ into $S^{4}$ (see do Carmo and Wallach [7]) and project stereographically into $R^{4}$. The reason this is so is that the property that the ellipse is a circle is a conformal invariant.

2. Atiyah and Lawson [2] have shown that an immersed surface in $S^{4}$ has the ellipse always a circle if and only if the canonical lift of the immersion map into the bundle of almost complex structures of $S^{4}$ is holomorphic. Holomorphic curves in this bundle can also be projected down to $S^{4}$ in order to obtain examples of surfaces in $S^{4}$ with the property that the ellipse is always a circle, hence giving equality in (1.1).

Corollary 3 (Ruh [12]). Let $M$ be homeomorphic to the 2-sphere $S^{2}$. If $f: M \rightarrow S^{4}$ is a minimal immersion with trivial normal bundle, then $f$ is totally geodesic.

REMARK 3. When $M$ is homeomorphic to the 2-sphere $S^{2}$ and $f$ : $M \rightarrow S^{n}(1)$ is minimal, then, by Theorem 1, the ellipse of curvture is always a circle. It is known that this circle degenerates to a point only at isolated points and that the plane that contains the circle extends to the singularities. Hence we have a 2-plane subbundle $P$ of the normal bundle; let $K^{*}$ be its intrinsic curvature (see $\$ 2$ for a definition).

In [3] Barbosa, shows that there is a large family of minimal immersions of the sphere. The following theorem shows that this is not so if we make some restrictions on the Gaussian curvature $K$ and the curvature $K^{*}$ of the immersion.

THEOREM 2. Let $f: M \rightarrow S^{n}(1)$ be a minimal immersion of a surface $M$ homeomorphic to the 2-sphere $S^{2}$ into the $n$-dimensional unit sphere $S^{n}(1)$. If $2 K \geq K^{*}$ at every point, then $K$ and $K_{N}$ are constant and $f$ is one of the generalized Veronese surfaces studied by Calabi [4] and do Carmo-Wallach [7].

${ }^{\dagger} \mathrm{S}$. T. Yau has informed us that he obtained the same result. 
REMARKs. 4. If $n=4$ then $K^{*}=K_{N}$, and, in this case, $f$ must be totally geodesic or a covering of the Veronese surface.

5. The conclusion in Theorem 2 that the curvatures $K$ and $K_{N}$ be constant holds actually for any surface with parallel mean curvature vector with the property that the ellipse is always a circle.

2. The ellipse of curvature. We are considering immersions $f: M \rightarrow$ $Q_{c}^{n}$ of surfaces into spaces of constant curvature $c$. Let $\nabla^{\perp}$ denote the covariant derivative associated to the induced Riemannian connection in the normal bundle $N$ of the immersion, and let $R^{\perp}$ denote its curvature tensor. If $\alpha: T M \times T M \rightarrow N$ denotes the second fundamental form and $A_{v}$ is the symmetric endomorphism of $T M$ defined by $\langle\alpha(X, Y), v\rangle=$ $\left\langle A_{v} X, Y\right\rangle$, where $\langle, \quad\rangle$ is the inner product in $T Q_{c}^{n}$, then, we recall the Ricci equation

$$
R^{\perp}(X, Y) u=\alpha\left(X, A_{u} Y\right)-\alpha\left(A_{u} X, Y\right)
$$

where $X$ and $Y$ are tangent and $u$ is a normal vector field along $f$. If $\left\{X_{1}, X_{2}\right\}$ is a tangent frame then, we denote $\alpha_{i j}=\alpha\left(X_{i}, X_{J}\right) ; i, j=1,2$ and we define $a \wedge b$ as the endomorphism

$$
a \wedge b(c)=\langle b, c\rangle a-\langle a, c\rangle b
$$

it is easy to see that

$$
R^{\perp}\left(X_{1}, X_{2}\right)=\left(\alpha_{11}-\alpha_{22}\right) \wedge \alpha_{12} .
$$

Also, if the mean curvature vector $H$ and the Gaussian curvature $K$ are defined by $H=\frac{1}{2}$ trace $\alpha$ and $d \omega_{12}=-K \omega_{1} \wedge \omega_{2}$, respectively, it is easy to see that

$$
4\|H\|^{2}=\left\|\alpha_{11}+\alpha_{22}\right\|^{2}, \quad K=\left\langle\alpha_{11}, \alpha_{22}\right\rangle-\left\|\alpha_{12}\right\|^{2}+c .
$$

An interesting notion that comes up in the study of surfaces in higher codimension is that of the ellipse of curvature defined as $\left\{\alpha(X, X) \in N_{p}\right.$ : $\langle X, X\rangle=1\}$. To see that it is an ellipse, we just have to look at the following formula, for $X=\cos \theta X_{1}+\sin \theta X_{2}$

$$
\alpha(X, X)=H+\cos 2 \theta \frac{\left(\alpha_{11}-\alpha_{22}\right)}{2}+\sin 2 \theta \alpha_{12} .
$$

So we see that, as $X$ goes once around the unit tangent circle, $\alpha(X, X)$ goes twice around the ellipse. Of course this ellipse could degenerate into a line segment or a point.

Facts. 1. The following properties are equivalent at a point of the immersed surface: (i) the ellipse degenerates into a line segment or a 
point, (ii) $\left(\alpha_{11}-\alpha_{22}\right) / 2$ and $\alpha_{12}$ are linearly dependent, (iii) $R^{\perp}=0$, and (iv) if $\left\{\boldsymbol{v}_{i}\right\}$ is an orthonormal normal frame, the second fundamental forms $A_{v_{i}}$ are simultaneously diagonalizable.

2. From (2.3) it follows that if $R^{\perp} \neq 0$ then $u=\left(\alpha_{11}-\alpha_{22}\right) / 2$ and $v=\alpha_{12}$ are linearly independent and we can define a 2-plane subbundle $P$ of the normal bundle $N$. This plane bundle inherits a Riemannian connection from that of $N$. Let $R^{*}$ be its curvature tensor and define its curvature $K^{*}$ by $K^{*}=\left\langle R^{*}\left(X_{1}, X_{2}\right) e_{4}, e_{3}\right\rangle$ or $K^{*} \omega_{1} \wedge \omega_{2}=-d \omega_{34}$, where $\left\{X_{1}, X_{2}\right\}$ and $\left\{e_{3}, e_{4}\right\}$ are orthonormal oriented bases of $T_{p} M$ and $P_{p}$, respectively. Now, if $\xi$ is perpendicular to $P$, then from (2.3), $R^{\perp}\left(X_{1}, X_{2}\right) \xi$ $=0$. Hence, it makes sense to define the normal curvature as

$$
K_{N}=\left\langle R^{\perp}\left(X_{1}, X_{2}\right) e_{4}, e_{3}\right\rangle
$$

where $\left\{X_{1}, X_{2}\right\}$ and $\left\{e_{3}, e_{4}\right\}$ are orthonormal oriented bases of $T_{p} M$ and $N_{p}$, respectively. If $T M$ and $P$ are oriented, then $K_{N}$ is globally defined. In codimension 2, $N=P$ and $K_{N}$ has a sign. In higher codimension, if $R^{\perp} \neq 0, P$ is globally defined and oriented if $T M$ is. In this case, it is shown in [1] that $\chi(P)=2 \chi(M)$ and, when $n=4, M$ must be a sphere $S^{2}$.

3. The area $A$ of the ellipse is given as $A= \pm(\pi / 2) K_{N}$ the sign being positive if the way the ellipse is traversed by $\alpha_{p}(X, X)-H_{p}$ coincides with the orientation of $P$. To see this, we observe that we can choose $\left\{X_{1}, X_{2}\right\}$ orthonormal such that $u=\left(\alpha_{11}-\alpha_{22}\right) / 2$ and $v=\alpha_{12}$ are perpendicular. When this happens, they will coincide with the semi-axes of the ellipse. If the parametrization of the ellipse coincides with the orientation of $N$, then $e_{3}=u /\|u\|$ and $e_{4}=v /\|v\|$ define a positively oriented normal frame and

$$
\begin{aligned}
A & =\pi\left\|\frac{\alpha_{11}-\alpha_{22}}{2}\right\|\left\|\alpha_{12}\right\| \\
& =\frac{\pi}{2}\left\langle\left(\alpha_{11}-\alpha_{22}\right) \wedge \alpha_{12}, e_{3} \wedge e_{4}\right\rangle \\
& =\frac{\pi}{2}\left\langle R^{\perp}\left(X_{1}, X_{2}\right) e_{4}, e_{3}\right\rangle=\pi / 2 K_{N} .
\end{aligned}
$$

Therefore, we obtain that

$$
K_{N}=\left\|\alpha_{11}-\alpha_{22}\right\|\left\|\alpha_{12}\right\| .
$$

The case when $\{u, v\}$ is negatively oriented follows similarly.

4. From (2.5) we can see that the center of the ellipse is the mean curvature vector $H$.

5. In codimension 2 , if the origin of the normal plane is inside or on the ellipse, then the Gaussian curvature $K<0$. If the origin is outside the 
ellipse, then $K<0, K=0$ or $K>0$ according to whether the angle subtended by the ellipse from the origin is bigger than $\pi / 2$, equal to $\pi / 2$, smaller than $\pi / 2$ (see Wong [14]).

\section{Proofs of theorems.}

Proof of Theorem 1. Let $\left\{X_{1}, X_{2}\right\}$ be an orthonormal frame at $p$; as we saw in $\$ 2$, we can choose $\left\{X_{1}, X_{2}\right\}$ such that $u=\left(\alpha_{11}-\alpha_{22}\right) / 2$ and $v=\alpha_{12}$ are the semi-axes of the ellipse. Hence, from (2.4) and (2.7) we have

$$
\begin{aligned}
0 & \leq\left(\left\|\alpha_{11}-\alpha_{22}\right\|-2\left\|\alpha_{12}\right\|\right)^{2} \\
& =\left\|\alpha_{11}-\alpha_{22}\right\|^{2}+4\left\|\alpha_{12}\right\|^{2}-4\left\|\alpha_{11}-\alpha_{22}\right\|\left\|\alpha_{12}\right\| \\
& =\left\|\alpha_{11}\right\|^{2}+\left\|\alpha_{22}\right\|^{2}+2\left\|\alpha_{12}\right\|^{2}-2 K-4\left|K_{N}\right|+2 c \\
& =\|\alpha\|^{2}-2 K-4\left|K_{N}\right|+2 c .
\end{aligned}
$$

On the other hand,

$$
\begin{aligned}
4\|H\|^{2} & =\left\|\alpha_{11}+\alpha_{22}\right\|^{2} \\
& =\left\|\alpha_{11}\right\|^{2}+\left\|\alpha_{22}\right\|^{2}+2\left\langle\alpha_{11}, \alpha_{22}\right\rangle \\
& =\left\|\alpha_{11}\right\|^{2}+\left\|\alpha_{22}\right\|^{2}+2\left\|\alpha_{12}\right\|^{2}+2 K-2 c \\
& =\|\alpha\|^{2}+2 K-2 c .
\end{aligned}
$$

Hence, by (3.1) and (3.2), it follows that

$$
\|H\|^{2}+c \geq K+\left|K_{N}\right|
$$

with equality if and only if $u=v$, i.e. the ellipse is a circle. Integrating (3.3) over $M$, we get

$$
\begin{aligned}
\int_{M}\|H\|^{2} d M+c \operatorname{Area}(M) & \geq \int_{M} K d M+\int_{M}\left|K_{N}\right| d M \\
& \geq 2 \pi \chi(M)+\left|\int_{M} K_{N} d M\right|
\end{aligned}
$$

with equality if and only if $K_{N}$ does not change sign and the ellipse is always a circle.

Now, we suppose that $M$ is homeomorphic to the 2-sphere $S^{2}$. Given isothermal parameters $\left\{x_{1}, x_{2}\right\}$ on a neighborhood of $M$ and putting 
$X_{i}=\partial / \partial x_{i} ; i=1,2$ and $z=x_{1}+i x_{2}$, we can see easily that

$$
\phi=\left(\|u\|^{2}-\|v\|^{2}-2 i\langle u, v\rangle\right) d z^{4}
$$

is a differential form of degree 4. In order to show that (3.4) is holomorphic, we use the following two equations that are obtained from the Codazzi equations (see Chen [5] or Ruh [12]).

$$
\nabla_{X_{1}}^{\perp} u+\nabla_{X_{2}}^{\perp} v=E \nabla_{X_{1}}^{\perp} H, \quad \nabla_{X_{2}}^{\perp} u-\nabla_{X_{1}}^{\perp} v=-E \nabla_{X_{2}}^{\perp} H .
$$

Now, we obtain the Cauchy-Riemann equations as follows

$$
\begin{aligned}
& X_{1}\left(\|u\|^{2}-\|v\|^{2}\right)=2\left\langle\nabla_{X_{1}}^{\perp} u, u\right\rangle-2\left\langle\nabla_{X_{1}}^{\perp} v, v\right\rangle \\
& \quad=2 E\left\langle\nabla_{X_{1}}^{\perp} H, u\right\rangle-2\left\langle\nabla_{X_{2}}^{\perp} v, u\right\rangle-2 E\left\langle\nabla_{X_{2}}^{\perp} H, v\right\rangle-2\left\langle\nabla_{X_{2}}^{\perp} u, v\right\rangle \\
& \quad=2 E\left(\left\langle\nabla_{X_{1}}^{\perp} H, u\right\rangle-\left\langle\nabla_{X_{2}}^{\perp} H, v\right\rangle\right)+X_{2}(-2\langle u, v\rangle)
\end{aligned}
$$

and, similarly,

$$
\begin{aligned}
X_{2}\left(\|u\|^{2}-\|v\|^{2}\right)= & -2 E\left(\left\langle\nabla_{X_{2}}^{\perp} H, u\right\rangle+\left\langle\nabla_{X_{1}}^{\perp} H, v\right\rangle\right) \\
& -X_{1}(-2\langle u, v\rangle) .
\end{aligned}
$$

Hence, $\phi$ is holomorphic if and only if

$$
\left\langle\nabla_{X_{1}}^{\perp} H, u\right\rangle-\left\langle\nabla_{X_{2}}^{\perp} H, v\right\rangle=0, \quad\left\langle\nabla_{X_{2}}^{\perp} H, u\right\rangle+\left\langle\nabla_{X_{1}}^{\perp} H, v\right\rangle=0 .
$$

In our situation, since $H$ is parallel, $\phi$ is holomorphic. Since the only holomorphic differential on the sphere $S^{2}$ is the constantly zero one, we get that $\|u\|=\|v\|$ and $\langle u, v\rangle=0$ from which we obtain that $u$ and $v$ are semi-axes of the ellipse and that they have the same length. Hence, we have equality in (1.1). If the codimension is equal to two, then $K_{N}$ is the curvature of the normal bundle and $\int_{M} K_{N} d M=2 \pi \chi(N)$ (see Little [10]), thus giving (1.2).

Proof of Corollary 1. Formula (1.3) is a direct application of (1.2). The area of $M$ is a multiple of $4 \pi$, because $\chi(N)$ is always an even number (in fact, it is twice the self intersection number of the immersion). Also, it is well-known that two such immersions are regularly homotopic if and only if their normal bundles have the same Euler characteristic (see Hirsch [8]).

Proof of Corollary 2. By fact 2, $\chi(M)=2, \chi(N)=4$, and (1.4) follows from (1.1).

REMARK 5. In the proof of Theorem 1 we saw that the ellipse is always a circle. Using isothermal parameters $z=x_{1}+i x_{2}$ in a neighborhood of $p \in M$, we obtain from equations (3.5) that the functions $\omega_{\alpha}(z)$ $=\left\langle u, e_{\alpha}\right\rangle-i\left\langle v, e_{\alpha}\right\rangle, \alpha=3, \ldots, n$ satisfy the condition of the theorem in 
Chern ([6], §4), where $\left\{e_{3}, \ldots, e_{n}\right\}$ is an orthonormal frame in a neighborhood of $p$. Hence, we can conclude that the ellipse degenerates into a point at isolated points only, and that at these points the "osculating plane" $P$ is still well defined. Hence $P$ is a globally defined oriented plane subbundle of the normal bundle $N$.

Proof of Corollary 3. By the above remark we know that the ellipse is always a circle and that it is either a point only at isolated points or always a point. Since, by fact $3, K_{N}$ is the area of the ellipse, we see that we can assume that $K_{N} \geq 0$. However, since $0=\chi(N)=$ $(1 / 2 \pi) \int_{M} K_{N} d M$, we must have $K_{N}=0$. Therefore the immersion is totally geodesic, since it is minimal.

Proof of Theorem 2. We want to show now that the radius $\lambda$ of the circle is a constant function. Let $\lambda_{0}$ be the maximum value of $\lambda^{2}$ on $M$. If $\lambda_{0}$ is identically zero then the immersion is totally geodesic. Assume $\lambda_{0}>0$ and consider the set $B=\left(\lambda^{2}\right)^{-1}\left(\lambda_{0}\right)$. Since $B$ is always closed, if we show that it is open in $M$, then $B=M$. Let $p$ be a point in $B$; we will show that $\Delta \log \lambda^{2} \geq 0$ in a neighborhood $U$ of $p$. But then, since the maximum is attained at $p, \lambda^{2}$ must be constant in $U$, proving that $B$ is open.

Since $\lambda^{2}(p) \neq 0$ and the immersion is minimal, for any orthonormal frame field $\left\{X_{1}, X_{2}\right\}$ tangent to $M$ in $U, e_{3}=\lambda^{-1} \alpha_{11}$ and $e_{4}=\lambda^{-1} \alpha_{12}$ define an oriented frame in $P$. Using the covariant derivatives for the second fundamental form $\alpha$ and the Codazzi equations, we have

$$
\begin{aligned}
X_{1} \lambda^{2}= & 2\left\langle\nabla_{X_{1}}^{\perp} \alpha\left(X_{1}, X_{2}\right), \alpha\left(X_{1}, X_{2}\right)\right\rangle \\
= & 2\left\langle\left(\tilde{\nabla}_{X_{1}} \alpha\right)\left(X_{1}, X_{2}\right), \alpha\left(X_{1}, X_{2}\right)\right\rangle \\
& +2\left\langle\alpha\left(\nabla_{X_{1}} X_{1}, X_{2}\right), \alpha\left(X_{1}, X_{2}\right)\right\rangle+2\left\langle\alpha\left(X_{1}, \nabla_{X_{1}} X_{2}\right), \alpha\left(X_{1}, X_{2}\right)\right\rangle \\
= & 2\left\langle\left(\tilde{\nabla}_{X_{2}} \alpha\right)\left(X_{1}, X_{1}\right), \alpha\left(X_{1}, X_{2}\right)\right\rangle \\
= & 2\left\langle\nabla_{X_{2}}^{\perp} \alpha\left(X_{1}, X_{1}\right), \alpha\left(X_{1}, X_{2}\right)\right\rangle-4\left\langle\alpha\left(\nabla_{X_{2}} X_{1}, X_{1}\right), \alpha\left(X_{1}, X_{2}\right)\right\rangle \\
= & 2\left\langle\nabla_{X_{2}}^{\perp} \alpha\left(X_{1}, X_{1}\right), \alpha\left(X_{1}, X_{2}\right)\right\rangle \\
& -4\left\langle\nabla_{X_{2}} X_{1}, X_{2}\right\rangle\left\langle\alpha\left(X_{2}, X_{1}\right), \alpha\left(X_{1}, X_{2}\right)\right\rangle \\
= & 2\left\langle\nabla_{X_{2}}^{\perp} \lambda e_{3}, \lambda e_{4}\right\rangle-4 \lambda^{2}\left\langle\nabla_{X_{2}} X_{1}, X_{2}\right\rangle \\
= & 2 \lambda^{2}\left(\omega_{34}\left(X_{2}\right)-2 \omega_{12}\left(X_{2}\right)\right) .
\end{aligned}
$$


Denoting by $J$ the complex structure of $M$, we have $J\left(X_{2}\right)=-X_{1}$, and we can write the above equation as

$$
d \lambda^{2} \circ J\left(X_{2}\right)=2 \lambda^{2}\left(2 \omega_{12}-\omega_{34}\right)\left(X_{2}\right) .
$$

Similarly, we obtain

$$
d \lambda^{2} \circ J\left(X_{1}\right)=2 \lambda^{2}\left(2 \omega_{12}-\omega_{34}\right)\left(X_{1}\right) .
$$

Hence, from (3.7) and (3.8) we obtain

$$
\begin{aligned}
\frac{1}{2} \Delta \log \lambda^{2} & =-\frac{1}{2} d\left(d \log \lambda^{2} \circ J\right) \\
& =-d\left(2 \omega_{12}-\omega_{34}\right)=2 K-K^{*} .
\end{aligned}
$$

Hence, under the conditions of the theorem we get that $\log \lambda^{2}$ is constant and consequently $\lambda^{2}$ is constant. Hence $K_{N}=2 \lambda^{2}$ and $K=1-2 \lambda^{2}$ are constant. Finally, by [7], $M$ is a generalized Veronese surface.

\section{REFERENCES}

1. A. C. Asperti, D. Ferus and L. Rodriguez, Surfaces with non-zero normal curvature tensor (preprint).

2. M. F. Atiyah and H. B. Lawson, Jr., In preparation.

3. J. L. Barbosa, On minimal immersions of $S^{2}$ in $S^{2 m}$, Trans. Amer. Math. Soc., 210 (1975), 75-106.

4. E. Calabi, Minimal immersions of surfaces in Euclidean spheres, J. Differential Geometry, 1 (1967), 111-125.

5. B. Y. Chen, Geometry of submanifolds, New York, M. Dekker (1973).

6. S. S. Chern, On the minimal immersions of the two sphere in a space of constant curvature, Problems in Analysis, Princeton Univ. Press (1970), 27-40.

7. M. do Carmo and N. Wallach, Representations of compact groups and minimal immersions into spheres, J. Differential Geometry, 4 (1970), 91-104.

8. W. M. Hirsch, Immersions of manifolds, Trans. Amer. Math. Soc., 93 (1959), 242-276.

9. T. Itoh, Minimal surfaces in 4-dimensional Riemannian manifolds of constant curvature, Kodai Math. Sem. Rep., 23 (1971), 451-458.

10. J. A. Little, On singularities of submanifolds of a higher dimensional Euclidean space, Ann. Mat. Pura Appl. 83 (1969), 261-335.

11. C. L. E. Moore and E. B. Wilson, Differential geometry of two-dimensional surfaces in hyperspaces, Proc. of the Academy of Arts and Sciences, 52 (1916), 267-368.

12. E. A. Ruh, Minimal immersions of 2-sphere in $S^{4}$, Proc. Amer. Math. Soc., 28 (1971), 218-222.

13. P. Wintgen, Sur l'inégalité de Chen-Willmore, C. R. Acad. Sc. Paris T., 288 (11 jui 1979) Serie A p. 993-995.

14. Y. C. Wong, Contributions to the theory of surfaces in 4-space of constant curvature, Trans. Amer. Math. Soc., 59 (1946), 467-507.

15. S. T. Yau, Submanifolds with constant mean curvature I, Amer. J. Math., 96 (1974), 346-366.

Received September 29, 1981 
UNICAMP-IMECC

13100, Campinas, S.P.

BRASIL

AND

IMPA

ESTRADA DA. CASTORINA, 110

JARDIM BOTÂNICO

22460, RIO DE JANEIRO-RJ.

BRASIL

Current address: University of California at Berkeley 
